\title{
Overnight orthokeratology is comparable with atropine in controlling myopia
}

\author{
Hui-Ju Lin ${ }^{1,2,3}$, Lei Wan ${ }^{1,2}$, Fuu-Jen Tsai ${ }^{1,2}$, Yi-Yu Tsai ${ }^{3}$, Liuh-An Chen ${ }^{1,3}$, Alicia Lishin Tsai ${ }^{3}$ and Yu-Chuen Huang ${ }^{1,2^{*}}$
}

\begin{abstract}
Background: Many efforts have been invested in slowing progression of myopia. Among the methods, atropine administration and orthokeratology (OK) are most widely used. This study analyzed the efficacy of atropine and OK lens in controlling myopia progression and elongation of axial length.

Methods: This retrospective study included 105 patients (210 eyes) who wore OK lenses and 105 patients (210 eyes) who applied $0.125 \%$ atropine every night during the 3 following period. Student $t$-test, linear regression analysis, repeated measure ANOVA, and Pearson's correlation coefficient were used for statistical analysis.

Results: The change in axial length per year was $0.28 \pm 0.08 \mathrm{~mm}, 0.30 \pm 0.09 \mathrm{~mm}$, and $0.27 \pm 0.10 \mathrm{~mm}$ in the OK lens group, and $0.38 \pm 0.09 \mathrm{~mm}, 0.37 \pm 0.12 \mathrm{~mm}$, and $0.36 \pm 0.08 \mathrm{~mm}$ in the atropine group for years 1,2 , and 3, respectively. Linear regression analysis revealed an increase in myopia of $0.28 \mathrm{D}$ and $0.34 \mathrm{D}$ per year, and an increase in axial length of $0.28 \mathrm{~mm}$ and $0.37 \mathrm{~mm}$ per year in the OK lens and atropine groups, respectively. Repeated measure ANOVA showed significant differences in myopia $(p=0.001)$ and axial length $(p<0.001)$ between the atropine and OK lens groups; in astigmatism, there was no significant difference in these parameters $(p=0.320)$. Comparison of increases in axial length in relation to baseline myopia showed significant correlations both in the OK lens group (Pearson's correlation coefficient, $r=0.259 ; p<0.001)$ and atropine group $(r=0.169 ; p=0.014)$. High myopia patients benefited more from both OK lenses and atropine than did low myopia patients. The correlation of baseline myopia and myopia progression was stronger in the OK lens group then in the atropine group.
\end{abstract}

Conclusions: OK lens is a useful method for controlling myopia progression even in high myopia patients.

Keywords: Atropine, Axial length, Cornea endothelium, Myopia, Orthokeratology

\section{Background}

Myopia is the one of the most common ocular disorders in the world. The prevalence of myopia is about $20 \%-$ $30 \%$ in North American, Australian, and European populations [1-3], and much higher $(40 \%-70 \%)$ in the Asian population [4-6], especially in China [7-9]. Myopia is an important public health problem because it is associated with increased risk for chorioretinal degeneration, retinal detachment, and other vision-threatening abnormalities $[10,11]$. Several therapeutic methods exist for the correction of myopia, such as corrective spectacles, contact lenses, keratorefractive surgeries, intraocular lenses (IOLs),

\footnotetext{
* Correspondence: irisluu2396@yahoo.com.tw

'Department of Medical Research, China Medical University Hospital, No. 2

Yuh Der Road, Taichung 404, Taiwan

${ }^{2}$ School of Chinese Medicine, College of Chinese Medicine, China Medical

University, Taichung, Taiwan

Full list of author information is available at the end of the article
}

and clear lens extraction [12-14]. However, possible sequel of keratorefractive surgeries of high refractive errors, such as glare, halo, and contrast sensitivity have been reported $[15,16]$. Therefore, prevention of high myopia is of utmost importance.

The nonselective muscarinic acetylcholine receptor (mAChR) antagonist, atropine, slows down myopia progression in a dose-dependent manner as compared with that in a placebo-treated group [17-19]. Atropine has proved useful both in animal studies and human clinical trials and is now widely used to control progression of myopia. However, myopia is never completely resolved. In atropine users, increasing intraocular pressure and photo-stress of the crystalline lens and retina are often concerned; photophobia and poor near vision are often to be confronted with. Moreover, rebound effect after atropine cessation had been mentioned before [20]. After

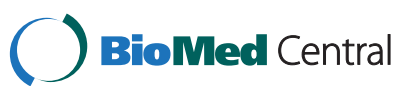


stopping treatment, eyes treated with atropine demonstrated higher rates of myopia progression compared with eyes treated with placebo. However, the absolute myopia progression after 3 years was significantly lower in the atropine group compared with placebo [20].

Orthokeratology (OK) uses specially designed rigid contact lenses to reshape the cornea in order to temporarily reduce or eliminate refractive error [21,22]. Modern OK using sophisticated contact lenses with a reversegeometry design can provide faster, larger, and more predictable refractive changes than OK lenses used in the original method introduced in the early 1960s [23]. Overnight OK lenses can decrease the patient's need to wear contact lenses or spectacles in the daytime by providing acceptable vision for normal routine activity. The presumed mechanisms of OK lens-induced myopic reduction include central corneal flattening, thinning of the central corneal epithelium $[24,25]$, thickening of the mid-peripheral cornea, and peripheral vision myopic shift [26]. However, several studies report that OK lenses increase higher-order aberrations of the cornea and decrease contrast sensitivity $[27,28]$. Despite controversies related to safety issues, OK lens use is becoming increasingly popular [21].

High myopia is one of the major causes of legal blindness, and many efforts have been invested into slowing elongation of axial length and decreasing myopia progression. In this study, two of the currently most useful methods in controlling myopia are compared: OK lenses and $0.125 \%$ atropine. Refractive errors, axial length, and endothelium cell count were analyzed to determine the effects of the two groups in controlling myopia progression.

\section{Methods}

This is a retrospective cohort study for a three years period, the patients using atropine or OK lens were grouped according to the selection of the patients themselves; no special recommendations were done in our department. The benefits and possible defects of the two methods were all informed to the patients and their families. Including photophobia, poor near vision and the risk of increasing intraocular pressure (IOP) might confront when using atropine; the risks of unstable vision in the daytime, glare at night and risks of keratitis in $\mathrm{OK}$ lens user. At the time of treatment, the patients and their families understood the different methods for treating myopia and selected the method themselves. Patients with complete clinical data during the study period (3 years, from March 2009 to March 2012) and undergone full and regular examinations were included in this study. All participants had a visual acuity with distance correction of $0.1 \operatorname{logMAR}(20 / 25)$ or better. Landolt C ETDRS Distance Chart was used. The UCVA (uncorrected visual acuity) and BCVA (best corrected visual acuity) were all measured between 2 and 4 pm for each patient. We used alpha of 0.05 , power of $80 \%$, and the sample size estimated was approximately 105 subjects for each group, the patients with former ID number were included. We selected the first 210 patients (105 atropine and $105 \mathrm{OK}$ lens) met the inclusion criteria and who visited our department had smaller ID number in our hospital; a total of 105 patients who used OK lens and 105 patients who used $0.125 \%$ atropine (Wu-Fu pharmaceutical Cc., Inc., YiLan, Taiwan) every night before sleep. Previous study of $\mathrm{Wu}$ et al. had proved that low concentration atropine is effective in controlling the progression of myopia [29], 0.125\% atropine was selected in the control group because that $0.125 \%$ atropine is the lowest concentration of atropine available and marketed in our country now. Their ages ranged from 7 to 17 years and myopia ranged from 1.5 to $7.5 \mathrm{D}$. Patients received $0.125 \%$ atropine and did not discontinue the drugs for more then 10 days during the study period (3 years). The study was approved by the ethics committee of China Medical University Hospital (Taichung, Taiwan) and was performed in accordance with the tenets of the Declaration of Helsinki for research involving human subjects. Informed consent was obtained from all participants. Comprehensive ophthalmic examinations were performed before treatment and at every visit. None of the participants had ocular insult or disease such as retinopathy, prematurity, neonatal problems, a history of genetic disease, and connective tissue disorders associated with myopia such as Strickler or Marfan syndromes. Clinical examinations included visual acuity, refraction error, slit lamp examination, ocular movements, intraocular pressure, and fundoscopy. Patients with organic eye disease, a history or evidence of intraocular surgery, and history of cataract were excluded from this study (Table 1). Non-cycloplegic subjective vision and cycloplegic objective refraction recorded at the visits before commencement of $0.125 \%$ atropine or OK lens treatment (baseline) and 1 ,

\section{Table 1 Inclusion and exclusion criteria}

\begin{tabular}{|c|c|}
\hline Exclusion criteria & Inclusion criteria \\
\hline Retinopathy & $\begin{array}{l}\text { Aged: } 7-18 \text { year-old } \\
\text { (average } 10 \pm 2.3 \text { year-old) }\end{array}$ \\
\hline Prematurity & $\begin{array}{l}\text { Myopia: } 1.5 \mathrm{D} \text { to } 7.5 \mathrm{D} \\
\text { (average } 4.25 \mathrm{D} \pm 1.5 \mathrm{D} \text { ) }\end{array}$ \\
\hline Neonatal problems & $\begin{array}{l}\text { Astigmatism: } 0 \mathrm{D} \text { to } 2.75 \mathrm{D} \\
\text { (average } 0.75 \mathrm{D} \pm-0.75 \text { ) }\end{array}$ \\
\hline History of genetic disease & $\begin{array}{l}\text { Follow up: } 6-40 \text { months } \\
(24 \pm 1.8 \text { months })\end{array}$ \\
\hline $\begin{array}{l}\text { Connective tissue (e.g. Strickler or } \\
\text { Marfan syndromes) }\end{array}$ & $\begin{array}{l}\text { Distance correction: } 0.1 \text { log MAR } \\
\text { (20/25) or better }\end{array}$ \\
\hline \multicolumn{2}{|l|}{ Organic eye disease } \\
\hline $\begin{array}{l}\text { Intraocular surgery (e.g. history } \\
\text { of cataract) }\end{array}$ & \\
\hline
\end{tabular}


2, and 3 years after were compared. Myopic diopter and axial length were also checked every year after discontinuing use of OK lenses for 3 weeks in the summer vocation between the semesters. The refractive error (in diopters [D]) of each individual was measured after administering one drop of cycloplegic drug (1\% mydriacyl; Alcon, Berlin, Germany). The data of the patients were detected each eye and the averages of the two eyes were used for analyzing; the differences of myopia degree between the two eyes over then $2 \mathrm{D}$ and astigmatism over then $1.5 \mathrm{D}$ were also excluded from this study. Individuals with myopia from 1.5 to $7.5 \mathrm{D}$ (average, $4.25 \pm 1.5 \mathrm{D}$ ) and astigmatism from 0 to $2.75 \mathrm{D}$ (average, $0.75 \pm 0.75 \mathrm{D}$ ) (negative cylinder was used in this study) were included in this study; cases of extreme high myopia (over 7.5 D) and astigmatism (over $2.75 \mathrm{D})$ were excluded. Auto-refraction (Autorefractor/ auto-keratometer [ARK 700A; Nikon, Tokyo, Japan]) was conducted for both eyes by experienced optometrists who were trained and certified in the study protocols. Refractive data, sphere (s), negative cylinder (c), and axis measurements were analyzed.

Patients who applied atropine ophthalmic eye drops received one drop of $0.125 \%$ atropine every night before sleep and wore glasses prescribed by a certified ophthalmologist and modified according to any refractive changes during the follow-up period. The OK lenses used in this study were 4-zone, reverse-geometry lenses (Emerald Lenses; Euclid Systems Corp., Herndon, VA, manufactured from Boston XO material; Polymer Technology Corp., Wilmington, MA) with a nominal Dk of $\left.100 \times 10^{-11} \mathrm{~cm}^{2} / \mathrm{s}\right)(\mathrm{mL} \mathrm{O} / \mathrm{mL} \cdot \mathrm{mmHg})$. The nominal central thickness of the lenses was $0.22 \mathrm{~mm}$, and the diameter was $10.4-11.0 \mathrm{~mm}$. The parameters of the lenses were varied to achieve good centration and good fluorescein pattern. After the lenses were dispensed, the patients were advised to wear them every night for at least 6-8 consecutive hours. In OK lens group, the patients with myopia over then $5.75 \mathrm{D}$ would use double reverse curves and dual geometric (DG) designs OK lens from Euclid Systems Corp. The patients of the 2 groups returned for examination every 3 months and underwent slit lamp examinations for any adverse events. The OK lens fit was evaluated at these visits. The first spectacles given to the patients in atropine group were when their UCVA worsen then $0.3 \operatorname{logMAR}$. OK lenses and spectacles were replaced if visual acuity was worse than $0.3 \log$ MAR during the follow-up.

Refraction, visual acuity, axial length, and corneal endothelium cell count obtained before initiation of the treatments were used as the baseline values; measurements were monitored every 3 months thereafter and they were also checked every year after discontinuing use of OK lenses for 3 weeks in the summer vocation between the semesters. That is, refractive error in $\mathrm{OK}$ group was measured 3 weeks after lens cessation 3 weeks for each patient after UCVA was measured. The axial length was evaluated using a noncontact optic biometric device (IOL Master; Carl Zeiss Meditec AG, Jena, Germany). On each occasion, 5 successive measurements were obtained, and their mean was used as a representative value. The measurements data were obtained by a well-trained examiner to decrease the errors induced by different examiners. Changes in axial length were evaluated prospectively and compared. Noncontact specular microscopy of the central corneal endothelium was performed with an SP-2000 specular microscope (Topcon Co, Tokyo, Japan), and endothelial photographs were taken. Subsequently, the Topcon IMAGEnet processing system (Topcon) was used to analyze these images. The boundaries of at least 100 cells per image were digitized, and the mean endothelial cell density, coefficient of variation of cell area, and percentage of hexagonal cells were calculated by semi-automatic mode.

Data are presented as ranges or means \pm standard deviation. A student $t$-test was used to compare the baseline conditions of the two groups. Linear regression, repeated measure ANOVA and Pearson's correlation coefficient analyses were performed to compare the refractive error at baseline and increased axial length. The more significant linear correlation and higher regression coefficient $(\beta)$ indicate the higher positive correlation. A $p$ value $<0.05$ represented significant in this study.

\section{Results}

In the OK lens group, 105 patients (53 males and 52 females) who successfully completed the 3 -year follow-up examinations were enrolled. Their ages ranged from 7 to 17 years (average, $11.82 \pm 1.25$ years). Sixteen (15.24\%) patients were aged 7-9 years, $70(66.67 \%)$ were aged 10-13 years, and 19 (18.83\%) were aged 14-17 years (Table 2). At baseline, their myopia ranged from 1.5 to $7.5 \mathrm{D}$ (average, $4.25 \pm 1.5 \mathrm{D}$ ), and astigmatism ranged from 0 to $2.75 \mathrm{D}$ (average, $0.75 \pm 0.75 \mathrm{D}$ ); $\log \mathrm{MAR}$ uncorrected visual acuity (UCVA) was between 0.20 and $1.40 \log$ MAR (mean, $0.80 \pm 0.45$ ), and axial length ranged from 22.05 to $27.05 \mathrm{~mm}$ (mean, $24.12 \pm 1.25 \mathrm{~mm}$; Table 2). In the atropine group, 105 patients (53 males and 52 females) used $0.125 \%$ atropine every night throughout the 3 -year followup (Table 2). Their ages ranged from 7 to 17 years (average, $11.12 \pm 1.68$ years). Twenty-three $(21.91 \%)$ patients were aged 7-9 years, 70 (66.67\%) were aged $10-13$ years, and $12(11.41 \%)$ were aged 14-17 years. Among the 105 subjects, 90 (90.5\%) patients required spectacles to perform daily activities. At baseline, their myopia ranged from 1.5 to $7.5 \mathrm{D}$ (average, $4.0 \pm 1.75 \mathrm{D}$; Table 2) and astigmatism was between 0 and $2.75 \mathrm{D}$ (average, $0.5 \pm 0.75 \mathrm{D}$; Table 2); UCVA ranged from 0.10 to $1.40 \log$ MAR (mean, $0.81 \pm 0.28$; Table 2), and axial length ranged from 21.12 
Table 2 Baseline data of patients in the OK lens and atropine group

\begin{tabular}{llll}
\hline & OK $^{\#}$ (mean \pm SD) & Atropine (mean \pm SD) $^{\text {p value }}$ \\
\hline Age, y/o & $11.82 \pm 1.25$ & $11.12 \pm 1.68$ & 0.745 \\
Sex, M/F & $1: 0.99$ & $1: 098$ & 0.987 \\
Myopia (D ${ }^{\text {d }}$ & 1.5 to $7.5(4.25 \pm 1.5)$ & 1.5 to $7.5(4.0 \pm 1.75)$ & 0.975 \\
Astigmatism (D) & 0 to $2.75(0.75 \pm 0.75)$ & 0 to $2.75(0.5 D \pm 0.75)$ & 0.897 \\
UCVA $^{*}$ (log MAR $)$ & $0.8 \pm 0.45$ & $0.81 \pm 0.28$ & 0.982 \\
BCVA $^{\text {a }}$ (log MAR) & $0.1 \pm 0.015$ & $0.12 \pm 0.05$ & 0.876 \\
Axial length (mm) & $24.12 \pm 1.25$ & $24.23 \pm 1.35$ & 0.985 \\
\hline
\end{tabular}

\#OK: Orthokeratology.

$\$ 0.125 \%$ atropine.

*UCVA: Uncorrected visual acuity.

${ }^{a} B C V A$ : Best corrected visual acuity.

${ }^{b}$ log MAR: logarithm of the Minimum Angle of Resolution.

${ }^{\mathrm{d} D}$ : diopter.

to $27.23 \mathrm{~mm}$ (mean, $24.23 \pm 1.35 \mathrm{~mm}$; Table 2). At baseline, the 2 groups were comparable in terms of myopia $(p=0.975)$, astigmatism $(p=0.897)$, and axial length $(p=0.985)$ (Table 2). All demographic data on UCVA, axial length, age, and gender are listed in Table 2; there were no significant differences in all the baseline conditions between the groups.

Using linear regression analysis, we found that myopia increased by $0.28 \mathrm{D} \pm 0.18 \mathrm{D}$ and $0.34 \mathrm{D} \pm 0.21 \mathrm{D}$ per year in the OK lens group and the atropine group, respectively (Table 3 ). The change in myopia diopters per year was $0.29 \pm 0.31 \mathrm{D}, 0.27 \pm 0.24 \mathrm{D}$, and $0.28 \pm 0.31 \mathrm{D}$ in the OK lenses group, and $0.31 \pm 0.19 \mathrm{D}, 0.35 \pm 0.85 \mathrm{D}$, and $0.32 \pm 0.53 \mathrm{D}$ in the atropine group for years 1,2 , and 3, respectively (Table 4). The change in axial length per year was $0.28 \pm 0.08 \mathrm{~mm}$ and $0.37 \pm 0.09 \mathrm{~mm}$ in the OK lens group and the atropine group, respectively (Table 3). The change in axial length per year was $0.28 \pm 0.08 \mathrm{~mm}, 0.30 \pm 0.09 \mathrm{~mm}$, and $0.27 \pm 0.10 \mathrm{~mm}$ in the OK lens group, and $0.38 \pm 0.09 \mathrm{~mm}, 0.37 \pm$ $0.12 \mathrm{~mm}$, and $0.36 \pm 0.08 \mathrm{~mm}$ in the atropine group for years 1,2 , and 3 , respectively (Table 4 ). There are

Table 3 Predictors of myopia and astigmatism between atropine and OK lens groups by linear regression analysis

\begin{tabular}{|c|c|c|c|}
\hline $\begin{array}{l}\text { Dependent } \\
\text { variable }\end{array}$ & & $\begin{array}{l}\text { Regression coefficient } \\
(95 \% \text { confidence interval) }\end{array}$ & $\begin{array}{l}\text { Repeated } \\
\text { measure ANOVA } \\
\text { (between the } \\
\text { two groups) }\end{array}$ \\
\hline \multirow[t]{2}{*}{ Myopia } & OK lens ${ }^{\#}$ & $-0.28(-0.40 \sim-0.16)$ & $p=0.001$ \\
\hline & Atropine & $-0.34(-0.46 \sim-0.21)$ & \\
\hline \multirow[t]{2}{*}{ Astigmatism } & OK lens ${ }^{\#}$ & $\pm 0.02(0.05 \sim 0.03)$ & \\
\hline & Atropine & $\pm 0.01(0.05 \sim 0.02)$ & \\
\hline \multirow[t]{2}{*}{ Axial length } & Ok lens ${ }^{\#}$ & $0.28(0.20 \sim 0.36)$ & $p<0.001$ \\
\hline & Atropine & $0.37(0.29 \sim 0.44)$ & \\
\hline
\end{tabular}

"OK: Orthokeratology.

${ }^{\$} 0.125 \%$ atropine. also significant but weak differences comparing axial length and myopic degree each year and data are not showed. We also compared the changes in the group aged 10-13 years; the averaged changes of myopia were $0.29 \pm 0.21 \mathrm{D}$ and $0.34 \pm 0.31 \mathrm{D}$ in $\mathrm{OK}$ lens and atropine groups per years $(p=0.003)$. The averaged changes of axial length per years: OK lens and atropine groups were $0.29 \pm 0.11 \mathrm{~mm}$ and $0.37 \pm 0.12 \mathrm{~mm}$, respectively ( $p=0.0035)$. Astigmatism (analyzed using a negative cylinder) changed by $\pm 0.02 \mathrm{D}$ and $\pm 0.01 \mathrm{D}$ per year in the OK lens group and the atropine group, respectively (Table 3); the axis of astigmatism did not show significant changes during the study period in the 2 groups.

The change in mean cornea endothelium cell count was not significantly different between the OK lens and atropine group (change per year, $\pm 38 \mathrm{cell} / \mathrm{mm}^{2}$ and $\pm 30 \mathrm{cell} / \mathrm{mm}^{2}$; $p=0.785)$. The UCVA of the OK lens group was 0.2

Table 4 Increase of myopia, stigmatism and axial length in each year

\begin{tabular}{lll}
\hline Year/myopia (D) & OK lens $^{\#}$ & Atropine $^{\mathbf{5}}$ \\
\hline 1 & $0.29 \pm 0.31$ & $0.31 \pm 0.19$ \\
2 & $0.27 \pm 0.24$ & $0.35 \pm 0.25$ \\
3 & $0.28 \pm 0.31$ & $0.32 \pm 0.23$ \\
\hline Year/astigmatism (D) & OK $^{\#}$ & Atropine $^{\mathbf{5}}$ \\
\hline 1 & $\pm 0.08 \pm 0.11$ & $\pm 0.03 \pm 0.02$ \\
2 & $\pm 0.08 \pm 0.42$ & $\pm 0.09 \pm 0.12$ \\
3 & $\pm 0.12 \pm 0.35$ & $\pm 0.11 \pm 0.16$ \\
\hline Year/axial length (mm) & OK lens $^{\#}$ & Atropine \\
\hline 1 & $0.28 \pm 0.08$ & $0.38 \pm 0.09$ \\
2 & $0.30 \pm 0.09$ & $0.37 \pm 0.12$ \\
3 & $0.27 \pm 0.10$ & $0.36 \pm 0.08$ \\
\hline
\end{tabular}

"OK: Orthokeratology.

${ }^{\$} 0.125 \%$ atropine. 
logMAR (20/30) to -0.1 logMAR (20/16), and BCVA of the atropine group was $0.1 \log$ MAR $(20 / 25)$ to $-0.1 \log$ MAR (20/16) at 2 and 4 pm, respectively.

To understand the relationship between the refractive error at baseline and increased axial length, Pearson's correlation coefficient was employed. Significant correlation was found between these parameters in the OK lens group (Pearson's correlation coefficient; $r=0.259, p<0.001$ ) as well as in the atropine group $(r=0.169, p=0.014$; Figures 1 and 2). The effect of decreasing the progression of axial length was more pronounced in high myopia patients than in low myopia patients for both groups. The regression coefficient $(\beta)$ was higher in the OK lens group than in the atropine group ( $\beta=0.060$ [OK lens group] and 0.029 [atropine group], respectively).

In the OK lens group, the most common complication was allergic conjunctivitis; in 37 eyes (17.6\%), there was an uncomfortable feeling such as itching during daytime, watery discharge on awaking, and requirement of drugs for relieving the symptoms. The care solution of OK lens used were "Boston Conditioning Solution and Boston Cleaner" (Bausch \& Lomb Taiwan Ltd, Taiwan) or BIOCLEN Contact Lens Solution (BIOCLEN OPHTECS, Japan). Fifteen eyes (7.14\%) showed superficial keratitis, which improved 3-7 days after terminating the use of OK lenses, without the need for drug administration and the OK lens were used continuously after re-education the taking care methods of the lens. No other complications, including corneal ulcers, were noted. In the atropine group, 2 eyes (1 patient [0.095\%]) showed mild allergic blepharitis, which improved after topical application of anti-allergy medication. In OK lens group, the patients with myopia over then $5.75 \mathrm{D}$ would use double reverse curves and dual geometric (DG) designs OK lens from Euclid Systems Corp. Their UCVA were better then $0.2 \log$ MAR at $2-4 \mathrm{pm}$ and just 2 patients needed spectacles at evening for taking lessons after school, none of the other needed spectacles in daily life. The major complaints of atropine application were photophobia during the day (35\%), which could be resolved by photochromic lenses or sunglasses (72\%), and poor near visual acuity (12\%), which could be improved by multifocal lenses in most patients (96\%). As for the effects of multifocal lenses and photochromic lenses for delaying myopia progression are also important subjects but are not the main themes in this study and need another investigation. No other evident abnormality was noted during the treatment period.

\section{Discussion and conclusions}

Atropine is a well-known drug for treating myopia. In this study, OK lens is effective in slowing progression of myopia and increasing of axial length over a period of 3 years and is compatible with the effect of atropine. In our knowledge, this is the first paper compare the effect of atropine and Ok lens in controlling progression of myopia. In this study, we find that Ok lens is mild better then atropine in controlling axial length elongation and myopia progression. Besides, Ok lens users do not suffer photophobia and risk of inducing crowding of anterior

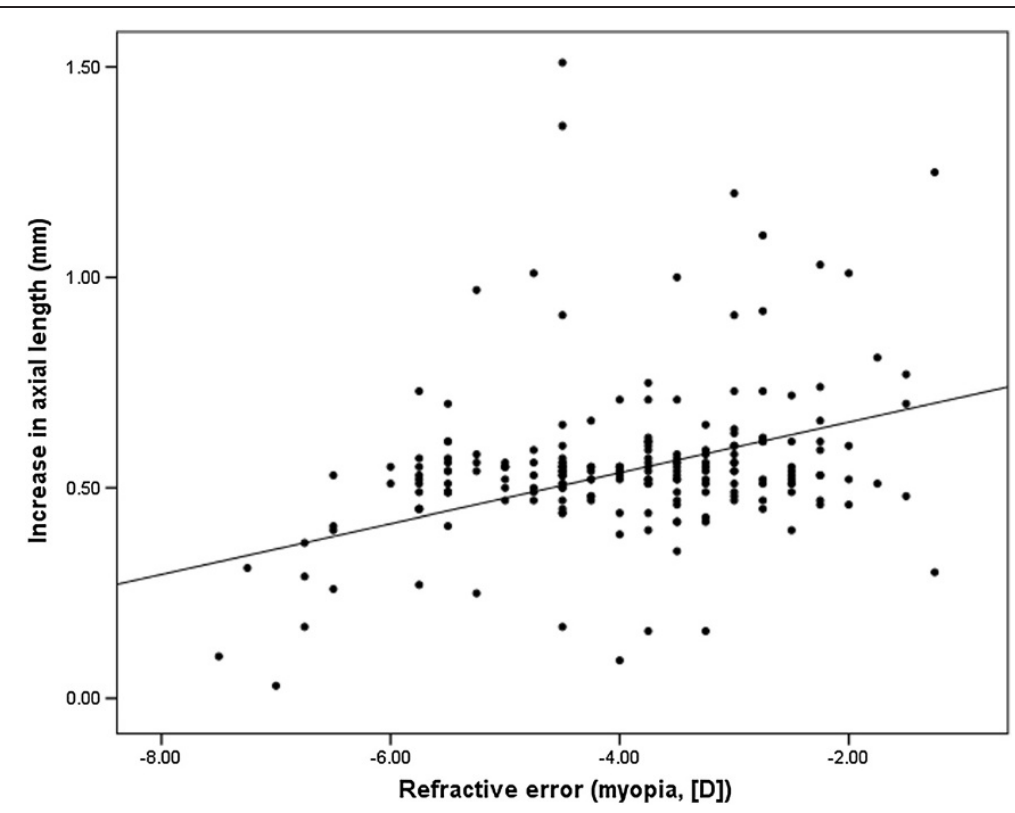

Figure 1 Increases in axial length $(\mathrm{mm})$ and refractive errors (myopia [D]) at baseline in the OK group. A significant correlation was found between the increases in axial length and spherical equivalent refractive errors (myopia [D]) at the baseline. Pearson's correlation coefficient: $r=0.259, p<0.001$ 


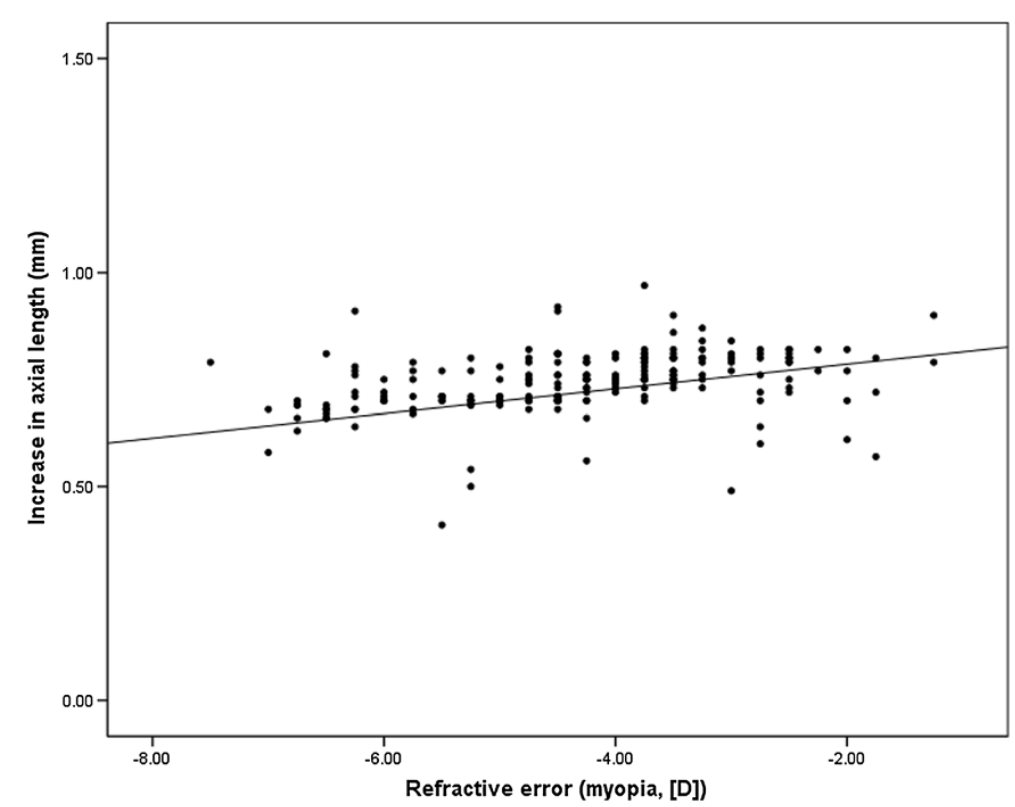

Figure 2 Increases in axial length $(\mathrm{mm})$ and refractive errors (myopia [D]) at baseline in the atropine used group. A significant correlation was found between the increases in axial length and spherical equivalent refractive errors (myopia [D]) at the baseline. Pearson's correlation coefficient: $r=0.169, p=0.014$.

chamber angle and increasing intraocular pressure (IOP) which may be induced by atropine. Moreover, the benefit of OK lens include that ceasing wearing glasses in daily life which bring convenience, better quality of life, no influence or near vision and somewhat good-looking. Nevertheless, there are still some drawbacks of OK lens, such as risk of cornea damage, infection and decreasing of cornea endothelium cell count; glare at night or vision decrease at evening are seen in OK lens users sometimes. There is no absolutely conclusion that OK lens or atropine is better, we just suggest that from the view of controlling myopia, Ok lens is useful methods.

The data presented in this study, the increase in axial length was $0.28 \pm 0.08 \mathrm{~mm}$ per year in the OK lens group versus $0.37 \pm 0.11 \mathrm{~mm}$ per year in the atropine group. In 2005, Cho et al. reported that axial length increased by $0.29 \pm 0.27 \mathrm{~mm}$ in the OK lens group and $0.54 \pm 0.27 \mathrm{~mm}$ in a control group treated with spectacles during a 2-year follow-up [30]. In 2009, Walline et al. reported similar findings; the mean increase in axial length after 2 years was $0.25 \mathrm{~mm}$ in the OK group and $0.57 \mathrm{~mm}$ in the control group [31]. In 2011, Kakita et al. obtained similar changes of $0.39 \pm 0.27 \mathrm{~mm}$ in the OK lens group versus $0.61 \pm 0.24 \mathrm{~mm}$ in the control spectacles group over 2 years [32]. The study of Walline et al. was performed with American patients, in whom the progression of myopia is reportedly slower than in the Asian population; this may explain the lesser increase in axial length in their study [31]. The results of Kakita et al. were obtained in Japan with an ethnic group similar to ours, and their results are similar to those of our study [32]. The increase in axial length in our OK lens group $(0.28 \pm 0.08 \mathrm{~mm}$ per year) was a little less than that reported by Cho et al. $(0.29 \pm 0.27 \mathrm{~mm}$ per year). The study of Cho et al. was performed in Hong Kong, in an area with high prevalence of myopia. Differences in the results may be because they used an ultrasonic A-mode device to measure axial length. In the present study, laser interferometry (IOLMaster; Carl Zeiss Meditec) was used to obtain noncontact measurements of axial length. This method has high reproducibility, and the non-contact procedure decreases the influences induced by compression of the cornea [33]. The increase in axial length in our atropine group was the smaller then the control groups of all the studies mentioned above. This can be understood by that $0.125 \%$ atropine is effective in decreasing myopia progression and axial length increase. Nevertheless, there were significant difference between the OK lens group and the $0.125 \%$ atropine group in quantity of axial length and myopic diopters. Moreover, there are data in our study worth pay attention, that is the standard deviation of atropine group in the second and third year is higher then in OK lens group, this might meant that the controlling myopia in this group is not very stable during the period.

The major limitation of our study is that the myopic diopter and axial length were checked every year after discontinuing use of OK lenses for only 3 weeks; this may not disturb the results of checking axial length but 3 weeks may not enough for the refractive error to completely recover to the baseline condition. However, 
patients in the OK lens group often relied on the OK lenses for their daily vision and could not discontinue use of the OK lens for 4-6 weeks [34], and 4-6 weeks has been proved to be sufficient for restoration of corneal curvature to the baseline condition so that the exact myopia diopter can be determined. This limitation is offset by the fact that the axial length, which is not influenced to a great extent by use of the OK lens, was also measured in this study, and a significant difference in axial length was found. Moreover, the included patients who completed the 3 years following-up without washout might be with satisfactory myopia control effect and lower or mild incidence of adverse effects; this induced the study biased towards successful cases. Nevertheless, the bias existed in both OK lens and atropine groups and cannot be neither avoided nor neglected.

Another limitation of our study is that age may influence the progression of myopia. A previous study demonstrated that myopia increased most remarkably at the age of 10-13 years; therefore, if we assessed only 10- to 13-year-old patients, the differences in myopic degree and axial length between the 2 groups were still observed, but to a lesser extent $(p=0.003$ and $p=0.0035$, respectively). However, the sample number was decreased after age stratification. Therefore, a larger study sample is required for future studies based on age stratification.

Allergic conjunctivitis happened in 37 eyes $(17.6 \%)$ of OK lens. The care solution of Ok lens used were "Boston Conditioning Solution and Boston Cleaner" (Bausch \& Lomb Taiwan Ltd, Taiwan) or BIOCLEN Contact Lens Solution (BIOCLEN OPHTECS, Japan). There was no evident difference that which solution will induce allergic conjunctivitis in our patients. The allergic conditions would subside after gave topic anti-histamine for 2 days without change care solutions. The allergic conjunctivitis might due to the warm and moist climate in our country. Patients selected the care solution at their own convenience. None of the patients had complains of the care solution.

Some of OK lens users exhibited UCVA greater than $0.2 \operatorname{logMAR}(20 / 30)$ or had obvious fluctuations in diurnal UCVA. These patients often had a flatter cornea curvature, irregular cornea surface, or tight eyelids. The limitations of OK lenses are obvious, and the tight eyelids of Asian subjects are a substantial concern. Although the new multiple-zone, reverse-geometry lenses have a better outcome, not all patients are satisfied with the OK lens. No severe corneal infection occurred in this study group; it is important to educate the patients and their families about maintenance of healthy habits and appropriate handling of OK lenses.

Patients with high myopia at baseline showed less severe increase in axial length than those with low myopia in both the OK lens and atropine groups. The linear correlation was more significant in the OK lens group then in the atropine group $(r=0.259$ versus $r=0.169)$. This phenomenon may occur because peripheral refraction changes are more evident in high myopia patients with OK lenses [32]. Myopic eyes usually have relative hyperopic defocus in the periphery, because the eye is elongated along the optic axis. Recent studies suggest that peripheral vision can influence axial length in human eyes, potentially altering the central refractive error and its development because of the emmetropization effect of eye growth. Conversion of relative peripheral hyperopia to relative peripheral myopia is a good method to limit the axial elongation that leads to myopia [26,35], and OK lenses appear to be an excellent option for achieving this objective. OK lenses appear to be a good tool to control high myopia. OK lenses create a small central zone and a smaller central visual field in high myopia patients comparing with lower myopia ones. At the same time, high myopia patients were with a greater area of the peripheral field remaining myopic. If changes of peripheral refraction are the primary reason for slowing progression of myopia, this would be expected to decrease axial length elongation especially in high myopia patients [36]. More human and animal studies are required to clearly test this hypothesis. However, clear central vision is essential for preventing defocus-inducing myopia.

The efficacy and safety of atropine is undoubted. Nevertheless, increasing intraocular pressure and photostress of the crystalline lens, retina, photophobia and poor near vision are often to be concerned in the patients using atropine. Low concentration atropine has proved useful in recent study that it can control myopia progression and decrease the side effects of high concentration atropine, low concentration atropine might an another good choice. OK lens with well care and hygiene may be one of the good policies to prevent progression of myopia and it does not just bring convenience for myopic patient to remove glasses in daytime. The combined use of OK lenses and atropine is a potential treatment for myopia progression and is being tested at our department. Hope this can give a new concept in delaying progression of myopia and can go a step further to resolve myopic problems.

\section{Abbreviations}

IOL: Intraocular lenses; mAChR: Muscarinic acetylcholine receptor;

OK: Orthokeratology; D: Diopters; logMAR: Logarithm of the minimum angle of resolution; UCVA: Un-corrected visual acuity; BCVA: Best corrected visual acuity.

\section{Competing interests}

All authors declare that they have no competing interests.

\section{Authors' contributions}

HJL has collected the data of the trial, designed the study and drafted the manuscript. LW participated in the design of the study and critically revised 
the manuscript. FJT collected the data and participated in the design of the study. YYT participated in its design and coordination. LAC had collected the data of the trial and participated in its design and coordination. ALT had collected the data. YCH performed the statistical analysis has given final approval of the version to be published. All authors read and approved the final manuscript.

\section{Acknowledgments}

This study was supported by National Science Council Taiwan (NSC 99-2314B-039-011-MY3). Moreover, we also thank the grants from China Medical University Hospital (DMR-100-097 and DMR-101-074, Taichung, Taiwan). We gratefully acknowledge Emily Hsieh and Chia Ming Wu from Department of Medical Genetics, China Medical University Hospital, Taichung, Taiwan for helping dealing experiment.

\section{Author details}

'Department of Medical Research, China Medical University Hospital, No. 2 Yuh Der Road, Taichung 404, Taiwan. ${ }^{2}$ School of Chinese Medicine, College of Chinese Medicine, China Medical University, Taichung, Taiwan.

${ }^{3}$ Department of Ophthalmology, China Medical University Hospital, Taichung, Taiwan.

Received: 10 August 2013 Accepted: 24 March 2014

Published: 31 March 2014

\section{References}

1. Midelfart A, Midelfart S: Prevalence of refractive errors among adults in Europe. Arch Ophthalmol 2005, 123:580

2. Kempen $\mathrm{JH}$, Mitchell $\mathrm{P}$, Lee KE, Tielsch JM, Broman AT, Taylor HR, Ikram MK, Congdon NG, O'Colmain BJ: The prevalence of refractive errors among adults in the United States, Western Europe, and Australia. Arch Ophthalmol 2004, 122:495-505.

3. Rose K, Smith W, Morgan I, Mitchell P: The increasing prevalence of myopia: implications for Australia. Clin Experiment Ophthalmol 2001, 29:116-120.

4. Sawada A, Tomidokoro A, Araie M, Iwase A, Yamamoto T: Refractive errors in an elderly Japanese population: the Tajimi study. Ophthalmology 2008, 115:363-370.

5. Wong TY, Foster PJ, Hee J, Ng TP, Tielsch JM, Chew SJ, Johnson GJ, Seah SK: Prevalence and risk factors for refractive errors in adult Chinese in Singapore. Invest Ophthalmol Vis Sci 2000, 41:2486-2494.

6. Pan CW, Wong TY, Lavanya R, Wu RY, Zheng YF, Lin XY, Mitchell P, Aung T, Saw SM: Prevalence and risk factors for refractive errors in Indians: the Singapore Indian Eye Study (SINDI). Invest Ophthalmol Vis Sci 2011, 52:3166-3173.

7. Liu HH, Xu L, Wang YX, Wang S, You QS, Jonas JB: Prevalence and progression of myopic retinopathy in Chinese adults: the Beijing Eye Study. Ophthalmology 2010, 117:1763-1768.

8. He M, Zheng Y, Xiang F: Prevalence of myopia in urban and rural children in mainland China. Optom Vis Sci 2009, 86:40-44.

9. Zhang M, Li L, Chen L, Wu J, Yang A, Chen C, Xu D, Lam DS, Sharma A, Griffiths S, Gao Y, Congdon N: Population density and refractive error among Chinese children. Invest Ophthalmol Vis Sci 2010, 51:4969-4976.

10. Moriyama M, Ohno-Matsui K, Shimada N, Hayashi K, Kojima A, Yoshida T, Tokoro T, Mochizuki M: Correlation between visual prognosis and fundus autofluorescence and optical coherence tomographic findings in highly myopic eyes with submacular hemorrhage and without choroidal neovascularization. Retina 2011, 31:74-80.

11. Ikuno $Y$, Jo Y, Hamasaki T, Tano Y: Ocular risk factors for choroidal neovascularization in pathologic myopia. Invest Ophthalmol Vis Sci 2010, 51:3721-3725

12. Kawesh GM, Kezerian GM: Laser in situ keratomileusis for high myopia with the VISX star laser. Ophthalmology 2000, 107:653-661.

13. Huang D, Schallhorn SC, Sugar A, Farjo AA, Majmudar PA, Trattler WB, Tanzer DJ: Phakic intraocular lens implantation for correetion of myopia: A report by the American academy of ophthalmology. Ophthalmology 2009, 116:2244-2258.

14. Pesando PM, Ghiringhello MP, Di Megilo G, Fanton G: Posterior chamber phakic intraocular lens for hyperopia: Ten-year follow-up. J Cataract Refract Surg 2007, 33:1579-1584.
15. Tuan KM, Liang J: Improved contrast sensitivity and visual acuity after wavefront-guided laser in situ keratomileusis: in-depth statistical analysis. J Cataract Refract Surg 2006, 32:215-220.

16. Oshika T, Okamoto C, Samejima T, Tokunaga T, Miyata K: Contrast sensitivity function and ocular higher-order wavefront aberrations in normal human eyes. Ophthalmology 2006, 113:1807-1812.

17. Shih YF, Hsiao CK, Chen CJ, Chang CW, Hung PT, Lin LL: An intervention trial on efficacy of atropine and multi-focal glasses in controlling myopic progression. Acta Ophthalmol Scand 2001, 79:233-236.

18. Cottriall $\mathrm{CL}$, Truong HT, McBrien NA: Inhibition of myopia development in chicks using himbacine: a role for M(4) receptors? Neuroreport 2001, 12:2453-2456.

19. Chia A, Chua WH, Cheung YB, Wong WL, Lingham A, Fong A, Tan D: Atropine for the treatment of childhood myopia: safety and efficacy of $0.5 \%, 0.1 \%$, and $0.01 \%$ doses (Atropine for the Treatment of Myopia 2). Ophthalmology 2012, 119:347-354.

20. Tong L, Huang XL, Koh AL, Zhang X, Tan DT, Chua WH: Atropine for the treatment of childhood myopia: effect on myopia progression after cessation of atropine. Ophthalmology 2009, 116:572-579.

21. Cho P, Cheung SW, Mountford J, White P: Good clinical practice in orthokeratology. Cont Lens Anterior Eye 2008, 31:17-28.

22. Swarbrick HA: Orthokeratology review and update. Clin Exp Optom 2006 89:124-143

23. Tahhan N, Du Toit R, Papas E, Chung H, La Hood D, Holden AB: Comparison of reverse-geometry lens designs for overnight orthokeratology. Optom Vis Sci 2003, 80:796-804.

24. Reinstein DZ, Gobbe M, Archer TJ, Couch D, Bloom B: Epithelial, stromal, and corneal pachymetry changes during orthokeratology. Optom Vis SCi 2009, 86:E1006-E1014

25. Zhong X, Chen X, Xie RZ, Yang J, Li S, Yang X, Gong X: Differences between overnight and long-term wear of orthokeratology contact lenses in corneal contour, thickness, and cell density. Cornea 2009, 28:271-279.

26. Kang $P$, Swarbrick $H$ : Peripheral refraction in myopic children wearing orthokeratology and gas-permeable lenses. Optom Vis Sci 2011 88:476-482.

27. Stillitano I, Schor P, Lipener C, Hofling-Lima AL: Long-term follow-up of orthokeratology corneal reshaping using wavefront aberrometry and contrast sensitivity. Eye Contact Lens 2008, 34:140-145.

28. Hiraoka T, Okamoto C, Ishii Y, Okamoto F, Oshika T: Recovery of corneal irregular astigmatism, ocular higher-order aberrations, and contrast sensitivity after discontinuation of overnight orthokeratology. $\mathrm{Br} J$ Ophthalmol 2009, 93:203-208.

29. Wu PC, Yang YH, Fang PC: The long-term results of using lowconcentration atropine eye drops for controlling myopia progression in schoolchildren. J Ocul Pharmacol Ther 2011, 27:461-466.

30. Cho P, Cheung SW, Edwards M: The longitudinal orthokeratology research in children (LORIC) in Hong Kong: a pilot study on refractive changes and myopic control. Curr Eye Res 2005, 30:71-80.

31. Walline JJ, Jones LA, Sinnott LT: Corneal reshaping and myopia progression. Br J Ophthalmol 2009, 93:1181-1185.

32. Kakita T, Hiraoka T, Oshika T: Influence of overnight orthokeratology on axial elongation in childhood myopia. Invest Ophthalmol Vis Sci 2011, $52: 2170-2174$

33. Olsen T, Thorwest M: Calibration of axial length measurements with the Zeiss IOLMaster. J Cataract Refract Surg 2005, 31:1345-1350.

34. Wu R, Stapleton F, Swarbrick HA: Residual corneal flattening after discontinuation of long-term orthokeratology lens wear in asian children. Eye Contact Lens 2009, 35:333-337.

35. Queirós A, González-Méijome JM, Jorge J, Villa-Collar C, Gutiérrez AR: Peripheral refraction in myopic patients after orthokeratology. Optom Vis Sci 2010, 87:323-329.

36. Charman WN, Mountford J, Atchison DA, Markwell EL: Peripheral refraction in orthokeratology patients. Optom Vis Sci 2006, 83:641-648.

\section{doi:10.1186/1471-2415-14-40}

Cite this article as: Lin et al:: Overnight orthokeratology is comparable with atropine in controlling myopia. BMC Ophthalmology 2014 14:40. 